\title{
CONTRIBUIÇÃO AO ESTUDO DA VASCULARIZAÇÃO ARTERIAL DO TESTICULO EM JUMENTOS DA RAÇA PEGA
}

\author{
CONTRIBUTION TO THE STUDY OF THE ARTERIAL VASCULARIZATION OF THE TESTICLES IN \\ DONKEY OF THE PEGA RACE
}

André Lulz Quagllatto SANTOS'; Arani Nancl Bomfim MARIANA; Vicente BORELLLI'; Marcos SILVA'; Renato Souto SEVFRINO'; Fredericu Ozanan Carneiro SILVA'; Sérgio Salazar DRUMOND'

\begin{abstract}
RESUMO
Estudou-se o componamento da artéria lesticular e scus ramos, bem como o número e distuibuiçāo dos vasos penetrantes, em 30 pares de testículos de jumentos dá raça Pega, adultos e procedentes de várias regiőes do Estado de Minas Gerais, mediante a análise de esquemas de modelos obtidos por corrosão após injeçåo de acelato de "vinyl". Observou-se que nestes animais essa antéria ofercce quatro arranjos vasculares diferentes, de acordo com sua divisån com predomínio de um ou outro dos vasos resultantes na irrigaçăo do órgāo. Quanto à distribuiçāo dos vasos penetrantes, por quadrante, em mediana, faz-se de maneira que nos testículos direitos o quadrante mais povoado é o craniomedial $(17,0)$, sendo seguido pelos quadrantes caudomedial e craniolateral $(15,5)$ e caudolateral $(15,0)$. Por outro lado, nos testículos esquerdos o quadrante caudolateral mostra o maior número de vasos penetrantes (18,5), seguido pelos quadrantes craniolateral $(17,0)$, craniomedial $(16,0)$ e caudomedial $(14,0)$. Indentificou-se a presença de anastomoses que ocorrem entre a anéria testicular e seus ramos ou entre os diferentes colaterais cedidos pela própria artéria. Relativamente ao número total de vasos penetrantes, encontrou-se em mediana, 66,5 e 66,0 vasos para os testículos direitos e esquerdos, respectivamente, o que estatisticamente năo é significante ao nível de $\alpha=0,05$, quando comparamos o número de vasos penetrantes dos testículos dircitos e esquerdos.
\end{abstract}

UNITERMOS: Anatomia, jumentos; Testículos; Artérias; Jumentos, raça Pega

\section{INTRODUÇÃO}

O jumento, por ser animal forte e ao mesmo tempo rústico, adaptou-se muito bem an clima brasileiro, principalmente à regiāo do nordeste do país, scrvindo como montaria e no transporte de cargas, constituindo-se às vezes na única opção do agreste. O Pega é um animal bem adaptado a essas finalidades nas condiçðes brasileiras por ser inteiramente nacional.

O conhecimento do médico veterinário no que diz respeito à anatomia e fisiologia dessa espécie toma-se indispensável, principalmente no que se refere à reproduçăo. Para tanto, os aspectos morfologicos dos testículos dos animais domésticos e silvestres, com especial referência ao sistema vascular, vêm sendo alvo de estudo dos pesquisadores.

Nesta oportunidade, propomos estudar o comportamento da artéria testicular e de seus ramos, bem como o número e distribuiçæo dos vasos penetrantes, em testículos de jumentos da raça Pega, buscando fornecer subsídios essenciais para o melhor conhecimento da anatomia testicular da espécie e ainda contribuir para o desenvolvimento da anatomia comparativa.

\section{MATERIAL E MÉTODO}

Para elaboração desta pesquisa, utilizaram-se 30 pares de testículos de jumentos da raça Pega, adultos, de diferentes e nảo conhecidas idades, procedentes de várias regióes do Estado de Minas Gerais e abatidos no Frigorífico AVANTE, no município de Araguari-MG.

Após o sacrifício dos animais, foram isolados os conjuntos constituídos pelos testiculos, epidídimos, funículos espermáticos e escroto, dos quais separaram-se os testículos com os respectivos epidídimos e funículos espermáticos, ainda envoltos pela lâmina visceral da túnica vaginal. Com o auxílio de paquimetro, registraram-se o comprimento (eixo craniocaudal) e largura (eixo lateromedial) dos 30 pares de testículos (Tab. 1).

Para o estudo da artéria testicular e seus ramos, foram abertas a túnica vaginal e a albugínca testicular, sob o corpo do epididimo ou lateralmente a ele, o que possibilitou mediante dissecação, isolar e canular a artéria testicular.

Após a lavagem dos vasos, foi injetada soluçāo de "vinyl"* corada em vermelho**, na quantidade de 4 a $6 \mathrm{ml}$ por peça.

1 - Professor Adjunto - Universidade Federal de Uberlândia

2 - Professor Assistente Doutor - Faculdade Medicina Veterinária e 7ootecria da USP

3 - Professor Titular - Faculadade Medicina Veterinária e Zootecnia da USP

- VMCHB - 1099. Union Carbide Corporation Chemical and Plastic. N. Y. - USA

* Laca Nitrocelulose vemelho molibdato. GLASSURIT DO BRASIL S/A. Indústrias de Tinus 
SANTOS, A.L.Q.; MARIANA, A.N.B.; BORELly, V.: SILVA, M.; SEVERINO, R.S.; SILVA, F.O.C.; DRUMOND, S.S. Contrnbuição ao estudo da vascularizaçío anterial do tescículo em jumentos da nạa Pega. Braz J. vet. Res. anim. Sct., São Paulo, v. 30, n. 2. p. 113-9. 1993.

O material foi mantido em água corrente cerca de 24 horas e depois submetido ao processo de corrosło em ácido sulfúrico a $30 \%$, por periodo de 72 a 96 horas, quando isolaram-se os modelos representativos da vascularizaçæo arterial do órgão.

De cada modelo realizaram-se desenho esquemático para análise e descriçăo dos resultados, registrando a divisăo da artéria testicular e seus ramos, bem como a origem e localizaçāo dos vasos penetrantes em relaçåo aos quadrantes craniomedial, craniolateral, caudomedial e caudolateral, obtidos mediante 0 traçado de planos perpendiculares entre si, sendo um entre as extremidades do órgão, delimitando as metades medial e lateral $(X)$ e outro no centro geométrico desse $(Y)$, demarcando as metades cranial e caudal (Fig. 1).

Para estudo estatístico, calculou-se a mediana, atinente ao número e localizaçåo dos vasos penetrantes e o teste "l" dc student para $\alpha=0,05$ (Tab. 2 e 3) e, para documentaçăo foram efctuadas algumas fotos (Fig. 2).

\section{RESULTADOS}

Nos jumentos da raça Pega, a artéria testicular apresenta trajeto sinuoso subalbugínico, através do qual alcança a margem epididimária do órgăo, próximo à extremidade capicata do mesmo e caminha sob o corpo do epidídimo, em posição medial ou lateral, acompanhando a margem epididimária em direçăo à extremidade caudata, até alcançar e contomar a mesma, oferecendo quatro diferentes arranjos vasculares (Grupos A, B, C e D).

Grupo A (Tab. 2 e 3): a artéria testicular emite de 4 a 11 ramos mediais e de 3 a 10 ramos laterais, anastomosando-se alguns deles com as artérias epididimárias, ao nivel da extremidade capitata do testículo, em 35 preparaçōes $(58,3 \%)$, isto é, 2 vezes $(3,3 \%)$ antes de envolver a extremidade caudata do órgåo ou, após contomá-la 33 vezes (54,9\%). Quanto ao número total de vasos penetrantes, observa-se como mediana e valores mínimo e máximo, respecuivamente, 65,0 (35 e 90), disuribuidos 21,0 (10 e 42) na face dorsal e 39,0 (16 e 66) na face ventral do órgão.

Grupo B (Tab. 2 e 3): a artéria testicular emite de 2 a 8 ramos adicionais e ao nível da metade do seu percurso, na margem livre do testiculo, divide-se em um ramo medial e outro lateral, em 12 preparaçðes $(20,0 \%)$. Verifica-se, nesses casos, em relaçăo aos ramos medial e lateral, vasos penetrantes, respectivamente, com mediana e valores mínimo e máximo 33,0 (13 e 64), distribuídos 10,5 (2 e 29) na face dorsal e 20,0 (10 e 35) na face ventral e em relaçăo aos ramos adicionais, vasos penetrantes com mediana e valores mínimo e máximo, respecuivamente de 35,0 (11 e 47), distribuidos 11,0 (4 e 19) na face dorsal e 23,0 (7 e 35) na face ventral.

Grupo C (Tab. 2 e 3): a artéria testicular, após contornar a extremidade caudata do testículo, fornece os ramos medial e lateral, de maneira a existir a predominância do ramo medial na vascularizaçăo arterial do órgăo, em 11 preparaçóes (18,3\%). Neste grupo, observam-se vasos penctrantes que apresentam, respectivamente como mediana e valores mínimo e máximo 55,0 (32 e 74), distribuídos 16,0 (4 e 35) na face dorsal e 32,0 (21 e 61) na face ventral.
Grupo D (Tab. 2 e 3): a artéria tescicular após contornar a extremidade caudata do testículo, fornece os ramos medial e lateral, de maneira a existir a predominância do ramo latera! na vascularìação arterial do órgão, em 2 preparaçós $(3,3 \%)$. Neste grupo registram-se vasos penetrantes com mediana e valores mínimo e máximo, respectivamente de 49,5 (45 e 54), distribuidos 16,0 (12 e 20) na face dorsal e 33,5 (33 e 34 ) na face ventral.

No estudo dos modelos da vascularizaçăo arterial do tesúculo do jumento da raça Pega, sem considerar os diferentes grupos, verifica-se, com base na mediana, que nos testículos direitos o quadrante craniomedial apresenta o maior número do vasos penetrantes $(17,0)$, seguido pelos quadrantes caudomedial $(15,5)$, craniolateral $(15,5)$ e caudolateral $(15,0)$, totalizando 66,5. Nos testiculos esquerdos, o quadrante caudolateral mostra o maior número de vasos penetrantes (18,5). seguidos pelos quadrantes craniolateral $(17,0)$, craniomedial $(16,0)$ e caudomedial $(14,0)$, wtalizando 66,0 (Tab. 4).

Em 9 preparaçðes (15,0\%), evidenciam-se anastomoses entre vasos que participam da nutriçåo do testiculo do jumento da raça Pega, isto é, em 4 peças do Grupo $A$, em 4 peças do Grupo B e em 1 peça do Grupo D.

\section{COMENTÁRIOS E CONCLUSÕES}

Para a artćria responsável pela nutriçāo do testículo, os autores têm utilizado diferentes denominaçoes, ou seja, artéria espermática ou grande testicular (BOSSI ${ }^{3}$, s.d.; BIMAR', 1888; LESBRE $^{17}$, 1923; LASSERRE; ARMINGAUD ${ }^{16}, 1934$ ), artéria grande testicular (MONGIARDINO20,1903), artéria espermática (BOURDELLE; BRESSOU`, 1938), artéria espermática interna (MANNU $\left.{ }^{19}, 1930\right)$ ) WOLFRAM ${ }^{24}, 1942$; KOCH's $^{\prime \prime}$ 1965; MORA ${ }^{21}, 1969$; SCHWARZE; SCHRÖDER ${ }^{25}$, 1972: ELLENBERGER; BAUM", 1977), artéria testicular interna (BRUNI; ZIMMERL's, 1977) e artéria testicular (HARRISON", 1949; JANTO SOVICOVA ${ }^{\prime 4}, 1969 ;$ IPPENSEN et al ${ }^{13}, 1972$; COLLIN', 1973; FEHLINGS ${ }^{9}, 1976$; GETTY ${ }^{10}$. 1981; VISINTIN ${ }^{27}$, 1985; DE VUONO?, 1986; LOPES ${ }^{14}$, 1987; MORETTII ${ }^{22}$, 1988; BOHÓRQUEZ MAHECHA $^{2}$, 1989; RODRIGUES ${ }^{24}$, 1989; PASSIPIERI ${ }^{23}$, 1990; SOUSA ${ }^{26}, 1991$ ), designação esta que ế a recomendada pela NOMINA ANATOMICA VETERINÁRIA (INTERNATIONAL COMMITTEE ON VETERINARY GROSS ANATOMICAL NOMENCLATURE'12, 1983), aqui uulizada. Ainda foram denominadas vasos penetrantes as artérias designadas ramos terminais (BOSSI ${ }^{3}$, s.d.; WOLFRAM" ${ }^{2}, 1942$ ), finos vasos (MONGIARDINO²0, 1903; MANNU ${ }^{19}, 1930$; SCHWARZE; SCHRÖDER ${ }^{23}, 1972$; GETTY ${ }^{10}, 1981$ ) artérias terminais (HARRISON", 1949), ramos testiculares profundos (BRUNI, ZIMMERL's, 1977) e pequenos ramos (ELLENBERGER; BAUM $\left.{ }^{8}, 1977\right)$.

Alguns autores como MONGIARDINO ${ }^{20}$ (1903); LESBRE"7 (1923); MANNU ${ }^{19}$ (1930) e GETTY ${ }^{10}$ (1981), apenas esclarecem que para o referido órgão esse vaso cede ramos, ou os definem como colaterais (BOURDELLE; BRESSOU ${ }^{4}, 1938$; MORA $^{21}$, 1969). Já BOSSI ${ }^{3}$ (s.d.) afirma que origina de 5 a 6 ramos colaterais, ou emite vasos para a face medial e face 
SANTOS, A.L.Q: MARIANA, A.N.B.; BORELLI, V.: SILVA, .M.; SFVERINO, R.S.; SILVA, F.O.C.; DRUMOND, S.S. Contribuiçăo ao estudo d. vascularizaçio anerial do testículo em jumentos da raca Pega. Braz. J. vet. Res. anim. Sci., São Paulo, v. 30, n. 2, p. 113-9, 1993.

lateral do órgăo (FEHLINGS\$, 1976), sem no entanto ater-se ao arranjo desses vasos.

De outra parte, IPPENSEN et al. ${ }^{13}$ (1972) e COLLIN ${ }^{6}$ (1973) encontraram com maior frequiência aqueles casos que aqui foram descritos caracterizando o Grupo A. Por outro lado, a descrição da artéria testicular, em alguns casos, dividindo-se dentro do cone arterioso, registrada por esses autores, correspondendo provavelmente aos ramos complementares, descritos por LOPES ${ }^{18}$ (1987) e BOHÓRQUEZ MAHECHA ${ }^{2}$ (1989), nåo foi aqui identificada. Em contrapartida, os casos $\mathrm{em}$ que esse vaso cedia inicialmente ramos adicionais para depois dividir-se nos ramos medial e lateral, năo foram mencionados por esse autores.

No jumento de raça Pega, assim como no suíno (MORETTI ${ }^{22}$, 1988) e no cavalo (BOHÓRQUEZ MAHECHA ${ }^{2}$, 1989) não ocorreu a equivalência de participação dos ramos medial e lateral, fato encontrado nos zebuinos (VISINTIN ${ }^{27}, 1985$ ), no jumento nordestino (DE VUONO', 1986), nos taurinos (LOPES $^{18}, 1987$ ) e nos ovinos (RODRIGUES ${ }^{24}, 1989$ ), nos búfalos (PASSIPIERI ${ }^{23}, 1990$ ) e nos caprinos (SOUSA ${ }^{26}$, 1991).

A presença de très ramos, medial, intermédio e lateral, oriundos da artéria testicular, foi verificada por RODRIGUES ${ }^{24}$ (1989) em ovinos e também por PASSIPIERI ${ }^{23}$ (1990) e SOUSA ${ }^{26}$ (1991) nos búfalos e caprinos respectivamente, embora estes autores tenham denominado esses vasos de ramos cranial, médio e caudal, respectivamente. Cabe salientar que, com exceção de RODRIGUES 24 (1989), os demais autores identificaram os ramos adicionais.

Relativamente ao número total de vasos penetrantes, registrado para o jumento da raça Pega, este apresenta-se superior ao encontrado para o cavalo e inferior ao evidenciado para o jumento nordestino. $\mathrm{O}$ que mostra que o número de vasos penctrantes não está, provavelmente, relacionado com o tamanho do testículo.

A presença de anastomoses entre os vasos que participam da nutrição do testículo foi evidenciada por todos os autores que estudaram este assunto com técnica semelhante à empregada aqui, exceção feita a VISINTIN ${ }^{27}$ (1985). Juntamente com a maioria das espécies estudadas, o jumento da raça Pega coloca-se numa posição intermediária, em número de anastomoses.

Finalmente, vale destacar que, na espécie em questão, năo se verificou diferença estatisticamente significante, para $\alpha=0,05$, quando confrontados os resultados obtidos nos tesúculos direitos com os dos esquerdos, tendo isto tamberm ocorrido para os taurinos, suínos, cavalos, ovinos, búfalos e caprinos.

Pelo que acaba de ser exposto, julga-se poder concluir que, nos jumentos da raça Pega:

1) o tamanho médio dos testículos é de $9,2 \mathrm{~cm}$ de comprimento e 3,6cm de largura;

2) a artéria testicular oferece diferentes arranjos vasculares, ou seja: emite número variado de ramos mediais e laterais $(58,3 \%)$; ramos medial e lateral, com predominância do pri- meiro ( $18,3 \%$ ) ou do segundo (3,3\%); ramos adicionais com subdivisăo em ramos medial e lateral ao nível da metade do seu percurso, na margem livre do testículo $(20,0 \%)$;

3) nos casos em que a artéria testicular cede os ramos lateral e medial, é comum nesta espécie, esse vaso fomecer antes ramos adicionais;

4) nos testículos direitos e esquerdos encontram-se, em mediana, respectivamente, 66,5 e 66,0 vasos peneuantes, nåo existindo entre eles diferença estatisticamente significante;

5) os quadrantes mais povoados, por ordem, à direita, saio: o craniomedial, o caudomedial, o craniolateral e o caudolateral, e à esquerda, o caudolateral, o craniolateral, o craniomedial e o caudomedial;

6) a face ventral do testículo concentra, mais comumente. maior número de vasos peneưantes em relaçăo à face dorsal;

7) anastomoses ocorrem em algumas preparaçðes (15,0\%).

\section{SUMMARY}

The course of the testicular artery and the number and distribution of its penetrating branches were studicd in 30 pairs of testicles of adults Pega donkeys originated from several regions of Minas Gerais (Brazil), in analysing the squematic draws from models obtenicd by corrosion after vinyl acctatc injection. In these animals it was noted that this artery shows four differents vascular arrangements according its division and the predominance in the organ blood supply showed by one or other of the resulting branches. The distribution of the penetrating branches per quadrant in median values, is made so that in the right testicles the most vascularized is the craniomedial $(17,0)$, followed by the caudomedial and the craniolateral $(15,5)$ and the caudolateral $(15,0)$. In the left testicle, the caudolateral quadrant showed the highest number of penetrating branches $(18,5)$, followed by the craniolateral $(17,0)$, craniomedial $(16,0)$ and the caudomedial $(14,0)$. Anastomosis between the testicular artery and its branches or between different collaterals which were originated from that artery were observed in $15.0 \%$ of the cases. The total number of penetrating branches in median values was respectively 66.5 and 66.0 to the right and to the left testicle. There were not significant statistical differences between the right tescicle and the left one. when the numbers of penetrating branches were compareted.

UNITERMS: Anatomy of donkeys; Testis; Arteries; Donkeys, Pega-breed

\section{REFERÊNCIAS BIBLIOGRÁFICAS}

01-BIMAR, M. Recherches sur la distribution des vaisseaux spermatiques chez divers mammiferes. C.R. Acad. Scl., Paris, v. 106, p.80-3, 1888.

02-BOHORQUEZ, MAHECHA, G.A. Contribuição ao estudo da vascularização arterial do testículo em cavalos (Equus caballus). Săo Paulo, 1989. Tese (Doutorado) - Faculdade de Medicina Veterinária e Zootecnia, Universidade de Såo Paulo. 
SANTOS, A.L.Q.; MARIANA, A.N.B.; BORELLI, V.; SILVA, M.; SEVERINO, R.S.; SILVA, F.O.C.; DRUMOND, S.S. Cuntribuiç̧ąo ao estudo da vascularização arterial do testículo em jumentos da rạ̧ Pega. Braz. J. vet. Res. anim. Sci., São Paulo, v. 30, n. 2. p. 113-9, 1993.

03-BOSSI, V. Angiologia. In: BOSSI, V.; CARADONNA, G.B.; SPAMPANI, G.; VARALDI, L.; ZIMMERL, U. Trattato dI anatomia veterinári̊. Milano, Francesco Vallardi, s.d.,v.2, p.210-3.

04-BOURDELLE, E.; BRESSOU, C. Anatomie régionale des animaux domestiques. 2.ed. Paris, J.B. Baillière, 1938. v.1, p. $724-5$.

05-BRUNI, A.C.; ZIMMERL, U. Anatomia degll animall domesticl. Milano, Franscesco Vallardi, 1977. v.2, p.171.

06-COLLIN, B. La vascularisation arteriélle du testicule chez le cheval. Anat. Histol. Embryol., v.2, p.46-53, 1973.

07-DE VUONO, L. Contribulçāo ao estudo da vascularlzação arterial do testículo em Jumento nordestino. São Paulo, 1986. Tese (Doutorado) - Faculdade de Medicina Veterinária e Zootecnia, Universidade de São Paulo.

08-ELLENBERGER, W.; BAUM, H. Handbuch de vergleichenden anatomle der Haustiere. 18 Anf. Berlin, Springer Verlag. 1977. p.696.

09-FEHLINGS, K. Korrosions-und röntgenanatomische Untersuchungen der Arteria testicularis von Katze, Hund, Schwein, Schaf, Rind und Pferd. Hannover, 1976. (Inaugural Dissertation) - Tierarztliche Hochschule.

10-GETTY, R. Sisson/Grossman anatomia dos animais doméstlcos. S. ed. Rio de Janeiro, Interamericana, 1981 v. 2, p.499, 562

11-HARRISON, R.G. The comparative anatomy of the blood-supply of mammalian testis. Proc. Zxxy. Soc. Lond, v.119, p.32544, 1949.

12-INTERNATIONAL COMMITTEE ON VETERINARY GROSS ANATOMICAL NOMENCLATURE. Nomina anutomica veterinaria. 3. ed. Ithaca, 1983.

13-IPPENSEN, E.; KLUG-SIMON, C.H.; KLUG, E. Der Verlauf der Blutgefäbe von Hoden des Pferdes im hinblik auf eine biopsiemoglichkeit. Zuchrhyg, v. 7. p. 35-45, 1972.

14-JANTO SOVICOVA. J. The intraorganic arterial system of the testis of rams, boars and stallions. Folla vet., v.13, n.3/4, p.26$31,1969$.

15-KOCH, T. Lehrbuch der Veterinär-Anatomie. Jena, Gustav Fischer, 1965. v.3, p.123.

16-LASSERRE, R.; ARMINGAUD, F. Anatomie des vaisseaux testiculaires chez le cheval et applications a la pathologie chirurgicale. Rev. vet. J. Méd-Vet., v.85, p.13-38, 1934.
17-LESBRE, F.X. Précis d'anatomle comparée des animaux domestiques. Paris, J.B. Baillière, 1923. v.2, p.102.

18-LOPES, P.R.B. Contribulçāo à estudo da vascularihação arterial do testiculo em bovinos (IBos taurus). São) Paulo, 1987. Dissertação (Mestrado) - Faculdade de Medicina Veterinária e Zootecnia, Universidade de São Paulo.

19-MANNU, A. Apparechio vascolare. In: /IMMERL.U. Trattato di anatomia veterinaria. Milano, Francesco Vallardi, 1930) v.2, p. 170-1.

20-MONGIARDINO, T. Trattato di anatomla topografica del mammiferi domesticl. Torino, Luigi Delgrosso, 1903. p.174.

21-MORA, G.R. Anatomla del caballos. Bogotá, Universidad Nacional de Colombia, 1969. v.1, p.43()-1.

22-MORETTI, A.S. Contribuiçāo ao estudo da vascularizaçāo do testículo de suínos. São Paulo, 1988. Tese (I)outorado) Faculdade de Mcdicina Veterinária e Zootecnia, Universidade de Sāo Paulo.

23-PASSIPIERI, M. Contribuição ao estudo da vascularizaçāo arterial do testículo de búfalos da raça Murrah (Bubalus buballs). Sāo Paulo, 1990). Tese (Doutorado) - Faculdade de Medicina Veterinária e Zootecnia, Universidade de Sảo Paulo.

24-RODRIGUES, C.A. Contribuição yo estudo da vascularizaçāo arterial do testículo em ovinos da raça Corriedale (Ovis aries). São Paulo, 1989. Tese (Duutorado) - Faculdade de Medicina Veterinária e Zootecnia, Universidade de São Paulo.

25-SCHWAR'ZE, E.; SCHRODER, L. Compendio de anatomla veterinaria. Zaragoza, Acribia, 1972. v.3, p.72-3: Aparato circulatorio y piel.

26-SOUSA. M.R.Q. Contribuiçāo go estudo da vascularizaçāo arterial do testículo em caprinos (Capra hircus-Linnaeus, 1758) da raça Bhuj Brasilleira. São Paulo, 1991. Dissertação (Mestrado) - Faculdade de Medicina Veterinária e Tontecnia, Universidade de Sảo Paulo.

27-VISINTIN, J.A. Contribuição ao estudo da vascularizaçāo arterial do testículo em bovinos da raça Nelore. São Paulo, 1985. Tese (Doutorado) - Instituto de Ciências Biomédicas. Universidade de São Paulo.

28-WOLFRAM, W. Zur Anatomie der arteria spermatic intema. Klin. Wschr., v.21, p.1126-7, 1942.

Recebido para publicaça em $26 / 10 / 92$ Aprovado para publicação em $05 / 12 / 93$ 


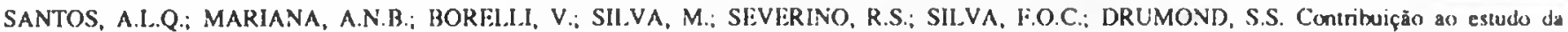
vascularizaçäo arterial do tesúculo cm jumentos da raça Pega, Bray. J. vet. Res. anim. Scí. São Paulo, v. 30, n. 2, p. 113-9, 1993.

TABELA 1

Comprimento e largura de testículos Direito (D) e Esquerdo (E) de jumentos da raça Pega. Sáo Paulo, 1992.

\begin{tabular}{|c|c|c|}
\hline OBS & $\begin{array}{c}\text { comprimento } \\
(\mathrm{cm})\end{array}$ & $\begin{array}{c}\text { largura } \\
(\mathrm{cm})\end{array}$ \\
\hline 010 & 9,8 & 4,1 \\
\hline $01 \mathrm{E}$ & 9.7 & 3,9 \\
\hline $02 \mathrm{D}$ & 9,2 & 3,9 \\
\hline $02 \mathrm{E}$ & 9.4 & 3.8 \\
\hline 030 & 8,6 & 3,0 \\
\hline $03 E$ & 8.8 & 3.4 \\
\hline $04 \mathrm{D}$ & 8,3 & 3.4 \\
\hline $04 \mathrm{E}$ & 8.5 & 3.5 \\
\hline $05 \mathrm{D}$ & 9.6 & 3.6 \\
\hline $05 \mathrm{E}$ & 9.0 & 3.4 \\
\hline $06 \mathrm{D}$ & 9,5 & 3.1 \\
\hline $06 \mathrm{E}$ & 9,1 & 3,3 \\
\hline $07 \mathrm{D}$ & 10.4 & 5.5 \\
\hline $07 \mathrm{E}$ & 9,1 & 4,8 \\
\hline $08 \mathrm{D}$ & 10,9 & 5.1 \\
\hline $08 \mathrm{E}$ & 10.7 & 5.0 \\
\hline $09 \mathrm{D}$ & 9.5 & 3.8 \\
\hline $09 \mathrm{E}$ & 9.1 & 3.5 \\
\hline $10 \mathrm{D}$ & 10,1 & 4.1 \\
\hline $10 \mathrm{E}$ & 9,6 & 3.9 \\
\hline 110 & 6.8 & 3.1 \\
\hline $11 \mathrm{E}$ & 7.2 & 3.4 \\
\hline $12 \bar{D}$ & 9,6 & 3,3 \\
\hline $12 \mathrm{E}$ & 9,7 & 3,1 \\
\hline $13 \mathrm{D}$ & 9.9 & 3,8 \\
\hline $13 \mathrm{E}$ & 10,1 & 3,8 \\
\hline $14 \mathrm{D}$ & 10,4 & 3.9 \\
\hline $14 \mathrm{E}$ & 9,7 & 3.5 \\
\hline $15 \mathrm{D}$ & 10,6 & 3,5 \\
\hline $15 \mathrm{E}$ & 10,4 & 3,8 \\
\hline $16 \mathrm{D}$ & 9,7 & 3.5 \\
\hline $16 \mathrm{E}$ & 9.9 & 3,8 \\
\hline $17 \bar{D}$ & 9,4 & 3,5 \\
\hline $17 \mathrm{E}$ & 9.5 & 4,0 \\
\hline $18 \mathrm{D}$ & 8.8 & 3,6 \\
\hline $18 \mathrm{E}$ & 8,7 & 3,7 \\
\hline $19 \mathrm{D}$ & 9.3 & 3.5 \\
\hline $19 \mathrm{E}$ & 9.4 & 3.4 \\
\hline 200 & 9,6 & 3,6 \\
\hline $20 \mathrm{E}$ & 9,1 & 3,3 \\
\hline $21 \mathrm{D}$ & 8,9 & 3.3 \\
\hline $21 \mathrm{E}$ & 9.1 & 3.5 \\
\hline $22 \mathrm{D}$ & 9.7 & 4.0 \\
\hline $22 \mathrm{E}$ & 9,5 & 3.9 \\
\hline 230 & 9,1 & 3.8 \\
\hline $23 \mathrm{E}$ & 9,0 & 3,5 \\
\hline $24 D$ & 9,5 & 3.4 \\
\hline $24 E$ & 9,2 & 3.3 \\
\hline $25 \mathrm{D}$ & 10.0 & 3,8 \\
\hline $25 \mathrm{E}$ & 9,6 & 3.4 \\
\hline $26 \mathrm{D}$ & 7,8 & 3,1 \\
\hline $26 \mathrm{E}$ & 8,2 & 3,3 \\
\hline 270 & 8,5 & 3.8 \\
\hline $27 \mathrm{E}$ & 8,3 & 4.0 \\
\hline $28 \mathrm{D}$ & 7.9 & 3.6 \\
\hline $28 \mathrm{E}$ & 8.4 & 3.9 \\
\hline 290 & 9,8 & 3,6 \\
\hline $29 \mathrm{E}$ & 9,6 & 3,5 \\
\hline $30 \mathrm{D}$ & 8.9 & 4.0 \\
\hline $30 \mathrm{E}$ & 8,7 & 3,8 \\
\hline módiz & 9.2 & 3,6 \\
\hline
\end{tabular}

TABELA 2

Número total de vasos penetrantes segundo sua modalidade. em mediana. nos diferentes grupos de arranjo da artéria testicular, em jumentos da raça Pega. Săo Paulo, 1992.

\begin{tabular}{lcccc}
\hline $\begin{array}{l}\text { Grupos } \\
\text { ramos }\end{array}$ & A & B & C & D \\
\hline Medial & 31,0 & 18,0 & 32,0 & 20,5 \\
Lateral & 34,0 & 14,5 & 20,0 & 29,0 \\
Adicionais & - & 16,5 & - & - \\
$\quad$ Mediais & & & & \\
Adicionals & & 18,5 & - & - \\
Laterals & & & \\
\hline
\end{tabular}

TABELA 3

Número total de ramos penetrantes por quadrante, em mediana, em relaçăo aos grupos de arranjo da artéria testicular em jumentos da raça Pega. Sáo Paulo, 1992

\begin{tabular}{lrrrr}
\hline Grupos & A & B & C & D \\
Quadrantes & & & & \\
\hline Craniomedial & 16,5 & 0,5 & 17,0 & 20.5 \\
Craniolateral & 15,5 & 0.5 & 16,0 & 15,5 \\
Caudomedial & 0.0 & 15,0 & 8,0 & 8,0 \\
Caudolateral & 0,0 & 14,0 & 16,0 & 5,5 \\
\hline
\end{tabular}

TABELA 4

Distribuiçáo dos vasos penetrantes por quadrante, em mediana, nos testiculos direito (D) e esquerdo (E) de jumento da raça Pega Sáo Paulo, 1992.

\begin{tabular}{lcc}
\hline Testiculos & D & E \\
Quadrantes & 17.0 & 16.0 \\
\hline Craniomedial & 15.5 & 17.0 \\
Craniolateral & 15.5 & 14.0 \\
Caudomedial & 15,0 & 18.5 \\
Caudolateral & \\
\hline
\end{tabular}




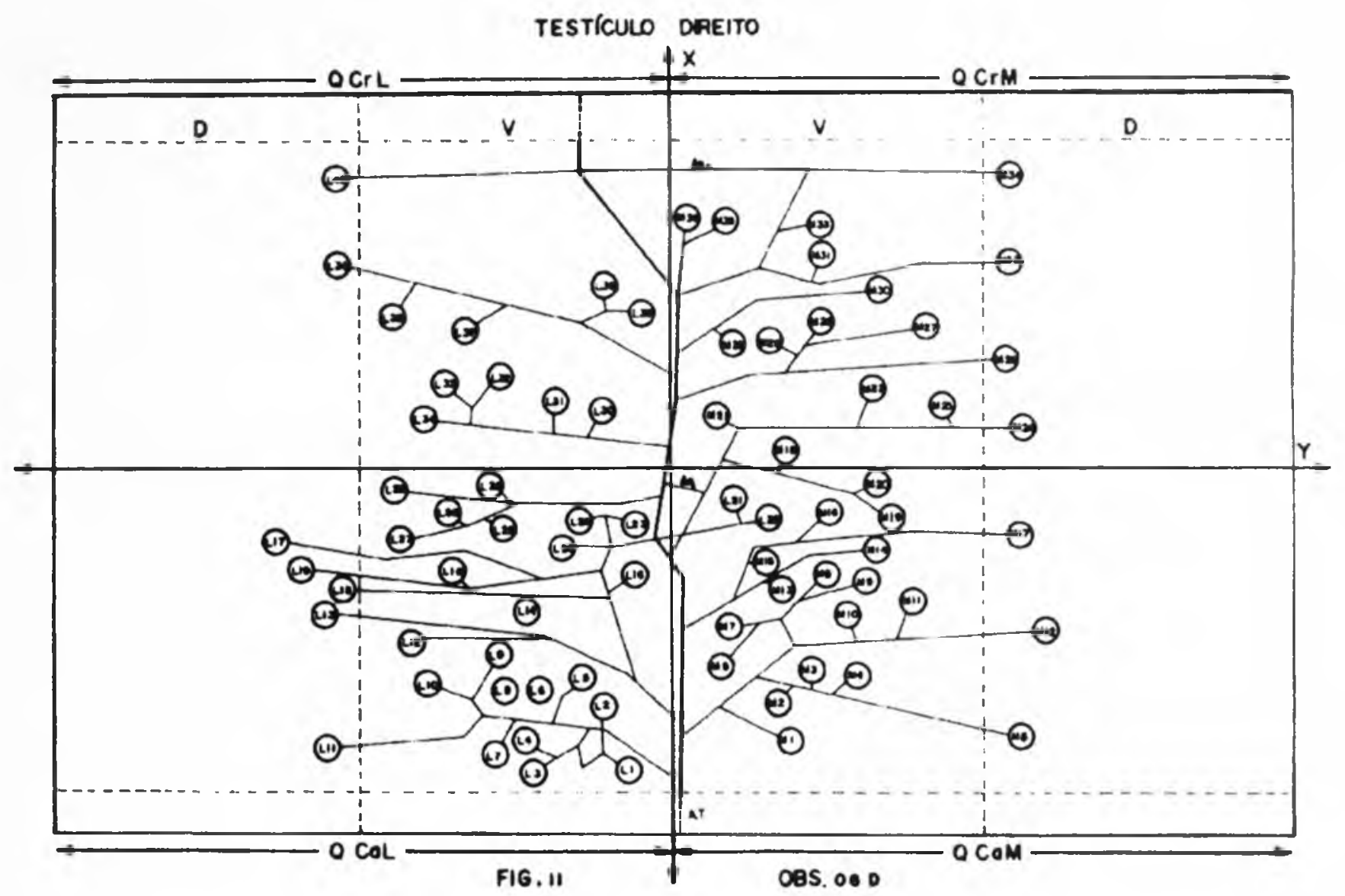

FIGURA 1

Desenho esquemálico do modelo em "vinyl", representando a antría testicular e seus ramos, assim como o número e a localizaçĭo dos vasus penee rantes nos quadrantes, no testículo de jumento da raça Pega.

AT. Antri Testicular

An - Anastomose

D Dorsal

V. Ventral

QCiM - Quadranle Craniomedial
OCrL - Oundrante Craniolseral QCaM - Quadrante Caudomodial QCaL - Quadranic Caudolmeral

M - Ramo Medial

L - Ramo Internal $x$ - eixo longitudinal do testículo $y$ - eixo transversal do testículo OBS - Observaçio (bes)

Fig. - Figura 


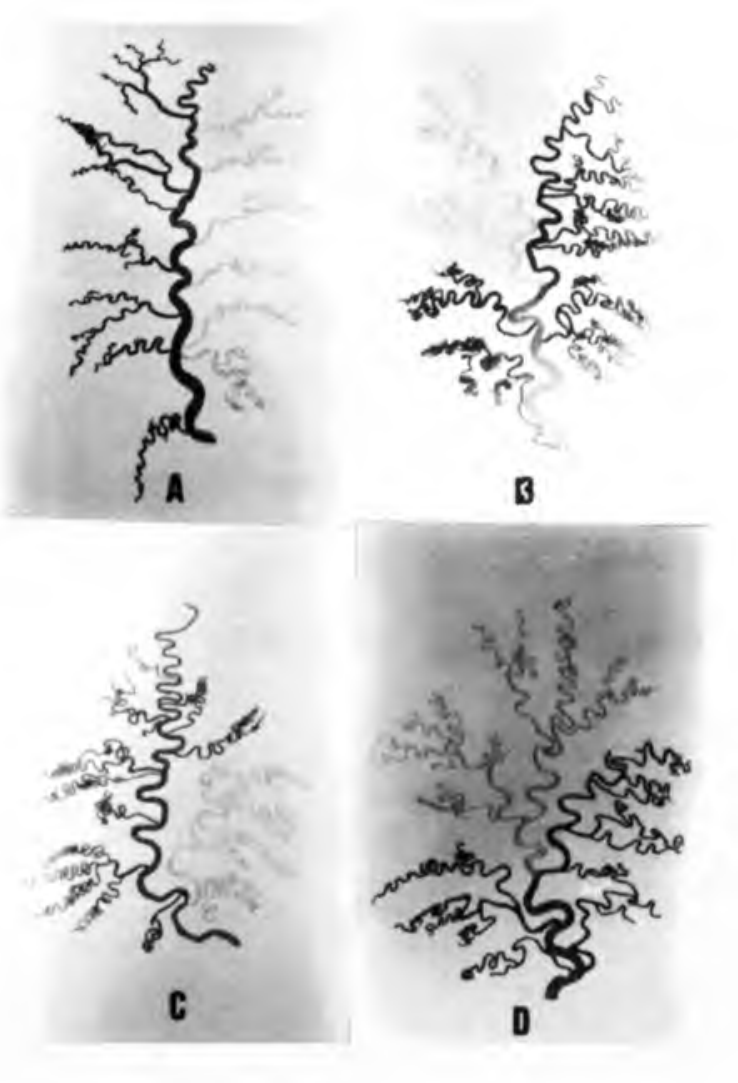

FIGURA 2

Fotografia dos modclos planificados em "vinyl", representativos dos quatro difierentes arranjos vasculares, dos iestículos de jumentos da raça Pega.

$$
\begin{aligned}
& A=\text { Grupo A } \\
& B=\text { Grupo B } \\
& C=\text { Grupo C } \\
& D=\text { Grupo D }
\end{aligned}
$$

